\title{
X-band transceiver module with a function of internal verification of receiving and transmitting channels
}

\author{
Maksim Karasev ${ }^{1, *}$, Alexander Dalinger ${ }^{1}$, Sergei Shatsky ${ }^{1}$, Sergei Shchyogolev ${ }^{1}$, \\ Andrey Adiatulin ${ }^{1}$, Dmitriy Zavyalov ${ }^{1}$, and Sergei Barskov ${ }^{1}$ \\ ${ }^{1}$ JSC “RPC “Istok ”named after A.I. Shokin”, Vokzalnaya, 2a, 141190 Fryazino, Russia
}

\begin{abstract}
The block diagram of the operability test of the receiving and transmitting channels as part of the transceiver module is described. The principle of operation of units verifying the operability of the receiving and transmitting channels is considered. The results of work of the developed circuit are analyzed.
\end{abstract}

\section{Introduction}

The transceiving modules (RTM) [1,2], provide different operating modes [3,4] and control of system parameters. Improving the electrical parameters and increasing the functionality of such devices make it possible to improve the overview of the scanned area, increase the range and resolution of the transceiver antenna.

To ensure fast control of the operability of the transceiver modules it is necessary to use built-in functional units allowing to obtain data on electrical parameters without involving additional equipment. Such units can be directional couplers with a power control system (PCS) forming feedback circuits, with the help of which the information about the channel operability is obtained when attenuators and phase shifters are controlled.

The principle of verifying the receiving and transmitting channels operability using the nodes of directional signal coupling is presented in the work.

\section{The transceiver module with a function of verification of receiving and transmitting channels operability}

The RTM block diagram is shown in figure 1.

The block diagram is composed of the following elements:

- a microwave switch of the transmitting channel (microwave Tx switch);

- a directional coupler of the transmitting channel (DC Tx);

- a directional coupler with a power control system of the receiving channel (DC with PCS Rx);

- a "receive/transmit" switch;

*Corresponding author: karasev.ms@gmail.com 


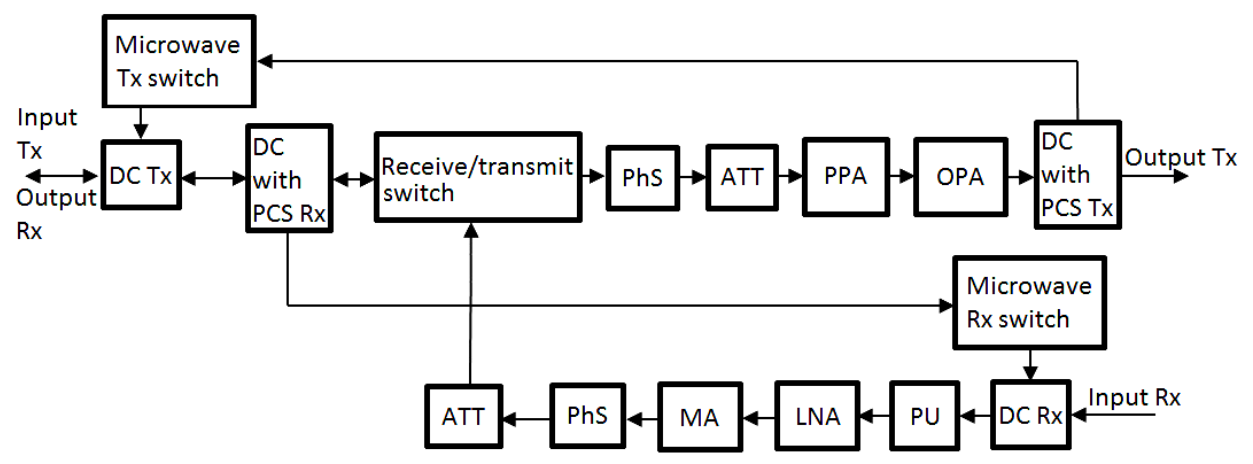

Fig. 1. The RTM block diagram with a function of verification of receiving and transmitting channels operability

- phase shifters $(\mathrm{PhS})$;

- attenuators (ATT);

- a power preamplifier (PPA);

- an output power amplifier (OPA);

- a directional coupler with a power control system of the transmitting channel (DC with PCS Tx);

- a microwave switch of the receiving channel (microwave Rx switch);

- a matching amplifier (MA);

- a low noise amplifier (LNA);

- a protective unit (PU);

- a directional coupler of the receiving channel (DC Rx).

The principle of verifying the transmitting channel operability.

A part of microwave power derived from the output power amplifier penetrates through the directional coupler with a power control system of Tx channel (DC with PCS Tx) to the input of Tx channel (when the microwave Tx switch is closed) through the directional coupler of Tx channel (DC Tx). A self-excitation of the transmitting channel arises due to positive feedback (PFB). The loop branch has a constant precalculated value relative to the path gain.

The introduction of attenuation into the path using attenuators (ATT) of Tx channel allows to remove the self-excitation of the path. The calculation by formula (1) makes it possible to calculate the Tx path gain and accordingly to confirm the operability of the transmitting channel.

$$
T x \text { gain }=\mid \text { feedback gain } \mid+\sum R \text { att }
$$

The channel self-excitation test is provided by a power control system on a detector diode, at the output of which voltage arises when the path is self-excited.

When the microwave Tx channel is closed self-excitation of the path may not occur as the phase characteristics do not meet the condition of $\Delta \varphi=0$. To initiate a self-excitation of the path it is necessary to introduce discharges of phase shifters before the appearance of self-excitation.

If there is no excitation of the Tx channel under all conditions of phase shifters and attenuators the Tx channel if faulty.

The principle of verifying the receiving channel operability.

A part of microwave power derived from the receiving channel output penetrates through the directional coupler with a power control system of Rx channel (DC with PCS $\mathrm{Rx}$ ) to the input of Rx channel (when the microwave Rx switch is closed) through the directional coupler of $\mathrm{Rx}$ channel (DC Rx). A self-excitation of the receiving channel arises due 
to positive feedback (PFB). The loop branch has a constant precalculated value relative to the path gain.

The introduction of attenuation into the path using attenuators (ATT) of Rx channels allows to remove the self-excitation of the path. The calculation by formula (2) makes it possible to calculate the path gain and accordingly to confirm the operability of the receiving channel.

$$
R x \text { gain }=\mid \text { feedback gain } \mid+\sum R \text { att }
$$

The channel self-excitation test is provided by a power control system on a detector diode, at the output of which voltage arises when the path is self-excited.

When the microwave Rx channel is closed self-excitation of the path may not occur as the phase characteristics do not meet the condition of $\Delta \varphi=0$. To initiate a self-excitation of the path it is necessary to introduce discharges of phase shifters before the appearance of self-excitation.

If there is no excitation of the Rx channel under all conditions of phase shifters and attenuators, the Rx channel if faulty.

\section{Measuring bench for verifying the operability of the transceiving module}

The measuring bench for verifying the operability of the module channels is shown in Figure 2 .

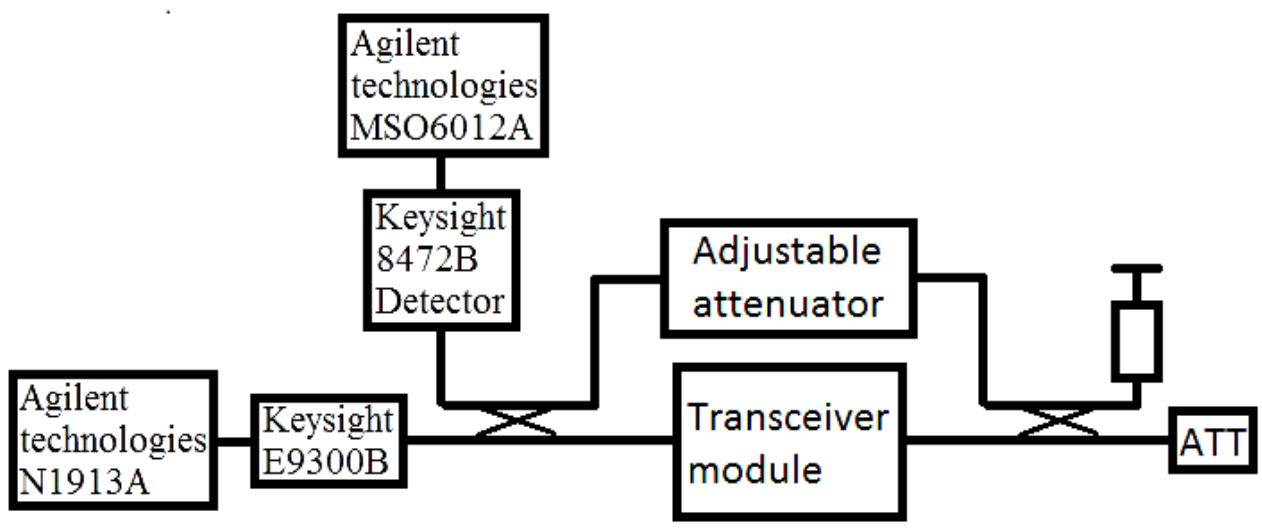

Fig. 2. The block diagram of the bench for imitating the function of verification of the module channels operability.

An adjustable attenuator is used to set the value of the feedback loop branch. The input and output of the module in the bench can vary depending on verification of $\mathrm{Rx}$ or $\mathrm{Tx}$ channels.

The results obtained during the experimental verification allow to conclude that the function of verifying the operability of transceiver channels is working correctly.

\section{Conclusions}

Using the developed diagram in modern transceiver modules makes it possible to obtain information about the operability of the channels of the transceiver module with high speed, accuracy, minimum of time and additional equipment. 


\section{References}

1. M.S. Karasev, V.A. Zhernovenkov, The design peculiarities of transmitter-receiver module "Abbat-I" using surface contact connector for transmitting microwave energy and low frequency signals of control and electric power supply, Collection of research $\&$ technical articles «Elektronnaya Tekhnika» Ser. 1 «SVCH-Tekhnika», Iss. 3(530), pp. 71-80 (2016)

2. M.S. Karasev, The methodology of parameter measurement of perspective active phased array transceiving modules using surface contact connector, Collection of research \& technical articles «Elektronnaya Tekhnika» Ser. 1 «SVCH-Tekhnika», Iss. 4(531), pp. 37-44 (2016)

3. M.S. Karasev, A.G. Dalinger, S.V. Shatsky, V.A. Zhernovenkov, X-band perspective multifunctional transmitter/receiver active phased array module, Collection of research $\&$ technical articles «Elektronnaya Tekhnika» Ser. 1 «SVCH- Tekhnika», Iss. 1(532), pp. 45-48 (2017)

4. M.S. Karasev, A.G. Dalinger, S.V. Shatsky, V.A. Zhernovenkov, E.A. Sinkova, S.A. Shchyigolev, Multifunctional AESA transceiver module of new generation, Proceedings of the conference «Electronics and microwave microelectronics-2018», pp. 239-242 (2018) 